Submitted on May 8, 2019 to the Notre Dame Journal of Formal Logic

Volume ??, Number ??,

\title{
The Formalities of Temporaryism without Presentness
}

\author{
Fabrice Correia and Sven Rosenkranz
}

\begin{abstract}
Temporaryism - the view that not always everything always existscomes in two main versions: presentism and expansionism (aka the growing block theory of time). Both versions of the view are commonly formulated using the notion of being present, which we, among others, find problematic. Expansionism is also sometimes accused of requiring extraordinary conceptual tools for its formulation. In this paper, we put forward systematic characterisations of presentism and expansionism which involve neither the notion of being present nor unfamiliar conceptual tools. These characterisations are full blown logics, each logic comprising an axiomatic proof system and an intuitive semantics with respect to which the system is both sound and complete.
\end{abstract}

\section{Introduction}

Following Timothy Williamson (2013, p. 4), let us call temporaryism the view that not always everything always exists, i.e. is something. Different ontologies can be temporaryist in this sense. According to standard conception, the formulation of such specific temporaryist ontologies requires appeal to the notion of presentness. The most prominent temporaryist ontologies on the market are presentism and expansionism (aka the growing block theory of time). Thus, presentism is standardly taken to essentially include the postulate that everything in time is present, and expansionism the postulates that everything in time is present or has been present in the past, and that something in time is not present. ${ }^{1}$

If the standard conception is correct, then temporaryist ontologies would seem vulnerable to recent objections by Williamson (2013) which challenge the suitability of the notion of presentness to cut much metaphysical ice. For instance, attempts to explicate the notion in terms of present existence or concreteness notoriously fail on this count, because on such an understanding of being present, even permanentist ontologies (according to which always everything always exists) can accommodate the claim that everything in time is present, including dinosaurs located at remote times.

Keywords: first-order temporal logic, presentism, growing block theory of time, presentness 
It is also often maintained, if less frequently so, that the formulation of expansionism needs to invoke extraordinary conceptual tools, e.g. a second notion of being present (Bourne 2002, Braddon-Mitchell 2004, Merricks 2006), non-standard tenses (Sider 2001), predications that are neither tensed nor untensed (Button 2006, 2007), or a special set of temporal operators (Tooley 1997). The need for such unfamiliar machinery would imply a considerable theoretical cost and would make expansionism seem less attractive from the start. But it would also show that the "logics" of presentism and expansionism differ more deeply than the difference in their characteristic, and mutually inconsistent, postulates would seem to suggest, since uncontroversially, presentism has no need for such extraordinary devices.

In this paper, we put forward precise characterisations of (versions of) presentism and expansionism which involve neither the notion of presentness nor the conceptual tools of the unfamiliar kinds just mentioned. ${ }^{2}$ Thus, besides standard Priorian temporal operators, truth-functional connectives, first-order quantifiers and an identity predicate, only two further pieces of vocabulary will be invoked, a predicate for times and a predicate for temporal location.

The characterisations will not consist in mere lists of principles, but will rather take the form of full blown logics, each logic comprising an axiomatic proof system and a corresponding adequate semantics. In each case, the semantics will be fairly simple and intuitive, and will therefore substantially add to the appeal that the corresponding system may independently have. Also, there will not be two characterisations, one for presentism and one for expansionism, but rather a set of characterisations for each view, each set corresponding to a number of natural variants of the relevant view.

Some of the ideas developed in this paper were first aired in Correia and Rosenkranz 2013 and 2015, and have then been substantially developed in Correia and Rosenkranz 2018. This latter work also puts forward a precise logical characterisation of each view involving a system and a corresponding semantic characterisation, but, importantly, these characterisations are formulated in a language which involves conceptual resources that are richer than those involved in the characterisations we propose in the present paper. The latter are accordingly philosophically more satisfactory than the former.

The plan is as follows. We first describe the languages in which the logics are to be formulated-the temporal languages (section 2). We then introduce a semantics which distinguishes between presentist models and expansionist models for such languages (section 3). After a digression on the expressive power of temporal languages interpreted in such models (section 4), we lay down a system Pres for presentism and a system Exp for expansionism (section 5). We then show that Pres is adequate with respect to the semantics of presentist models, and Exp with respect to the semantics of expansionist models (section 6). We subsequently discuss variants of Pres and $\operatorname{Exp}$ (section 7), and then move on to a discussion about the way temporal location should be characterised in the context of the logics discussed so far (section 8). We end up comparing the proposed characterisations of presentism and expansionism with those put forward in Correia and Rosenkranz 2013 and 2015 (section 9), and with those put forward in Correia and Rosenkranz 2018 (section 10). ${ }^{3}$ 


\section{Languages}

The languages in which the logics are to be formulated-the temporal languages, as we will call them-are standard first-order Priorean languages with identity, augmented with a special predicate for times and a special predicate for temporal location. More precisely, each such language has the following vocabulary:

- A countable set of predicates, including a set of distinguished predicates: the 1-place predicate $\mathrm{T}$ for times, and the 2-place predicates $=$ and $\mathrm{L}$ for identity and temporal location, respectively.

- A countably infinite set of variables, which we shall denote using the metavariables $x, y$, etc.

- A countable (possibly empty) set of constants, which we shall denote using the metavariables $a, b$, etc.

- The truth-functional connectives $\neg$ and $\vee$ and the existential quantifier $\exists$.

- The Priorean tense-logical operators $H$ (for 'Always in the past') and $G$ (for 'Always in the future').

- The brackets ( and ).

An element of the vocabulary is a term iff it is a variable or a constant. We shall use $m, n$, etc. as metavariables for terms. The formulas of a temporal language are defined in the usual way, and we shall adopt familiar notational conventions. On the intended interpretation, T $m$ will have to be understood as ' $m$ is a time', and $L m n$ as ' $m$ is located at time $n$ '. As is customary, we define

- $\forall x \phi$ as $\neg \exists x \neg \phi$,

- $P \phi$ ('Sometimes in the past, $\phi$ ') as $\neg H \neg \phi$,

- $F \phi$ ('Sometimes in the future, $\phi$ ') as $\neg G \neg \phi$,

- $S \phi$ ('Sometimes, $\phi$ ') as $P \phi \vee \phi \vee F \phi$,

- $A \phi$ ('Always, $\phi$ ') as $H \phi \wedge \phi \wedge G \phi$, and

- Em (' $m$ exists') as $\exists x(x=m)$ (where $x$ is the first variable distinct from $m$ according to some given enumeration of the variables).

In order to make clear which versions of presentism and expansionism we intend to characterise, we need to say a bit more about the intended interpretation of $\exists, T$ and $\mathrm{L}$. There are four points worth mentioning.

First, on its intended interpretation, $\exists$ expresses unrestricted quantification, but its range is nevertheless thought of as potentially sensitive to temporal standpoints: what there unrestrictedly is relative to a time need not be the same as what there unrestrictedly is relative to another time. We say 'potentially', because whether the range of the quantifier actually sometimes changes is a matter of substantive debate in temporal ontology: temporaryists hold that the range of the quantifier sometimes changes, while permanentists hold that it never does (see section 1).

Secondly, the concept of existence expressed by the defined predicate $E$ is therefore also potentially sensitive to temporal standpoints; yet existing at a time $t$, in the relevant sense of 'existing', should not be confused with being located at $t$, at least given our understanding of the concept of temporal location that $L$ is intended to express. Permanentists hold that always, everything always exists; but they do not thereby hold that Tim Williamson is located at all times: they hold (or at least should hold) that he is located only at the times when he is alive. Likewise, expansionistson our understanding of the expansionist view-hold that ever since he was born, 
Socrates has never ceased to exist; but they hold (or at least should hold) that he is located only at the times when he is alive, and hence that although he exists now, he is not located now. It is only presentists who could perhaps reasonably hold that 'exists at $t$ ' and 'is located at $t$ ' are always coextensional (but see the discussion of L4 and L5 in section 8).

Thirdly, given that $\exists$ is understood as unrestricted, the extension of $E$ at a time is intended to be the set of all the objects that there are relative to that time, whatever ontological categories they may belong to. Thus, in particular, not only do nonoccurrents such as people, planes and planets count as possibly within the extension of $E$, but the same holds also of occurrents such as lives, take-offs and climate changes, even though it is perhaps not customary to attribute existence at times to events and processes in ordinary English.

Finally, predicate $\mathrm{T}$ is intended to have in its extension only concrete times-e.g. "container times", as substantivalism would have it, or maximal fusions of contemporary instantaneous events, as some versions of relationism would have it-as opposed to so-called "ersatz times", i.e. abstract representations of what is going on at concrete times. It is very important to keep this point in mind, since on our understanding of the views, both presentism and expansionism hold that what times exist constantly varies with time, while it is not our intention to claim that these views cannot defend an ontology of permanently existing ersatz times. ${ }^{4}$

\section{Semantics}

We define a structure as a tuple $\langle\mathscr{T},\langle, D\rangle$, where

- $\mathscr{T}$ (times) is a non-empty set,

- $<$ (precedence) is a binary relation on $\mathscr{T}$ that is transitive and total, and

- $D$ (domain) is a function which assigns, to each $u \in \mathscr{T}$, a set $D(u)$ (the objects which exist at time $u$ ) such that $\bigcup_{u \in \mathscr{T}} D(u) \neq \varnothing$.

A structure $\langle\mathscr{T},\langle, D\rangle$ is said to be:

- proper iff $\mathscr{T}$ has at least two members, and for all $u \in \mathscr{T}, u \in D(u)$.

- presentist iff (c1) it is proper, (c2) for all $u, v \in \mathscr{T}, u<v \Rightarrow v \notin D(u)$, and (pres) for all $u, v \in \mathscr{T}, v<u \Rightarrow v \notin D(u)$.

- expansionist iff (c1) it is proper, (c2) for all $u, v \in \mathscr{T}, u<v \Rightarrow v \notin D(u)$, and (exp) for all $u, v \in \mathscr{T}, u<v \Rightarrow D(u) \subseteq D(v)$.

Three points are worth emphasising. First, given the requirement that $<$ be total, presentist structures satisfy the following condition: for all $u, v \in \mathscr{T}, u \in D(v) \Rightarrow$ $u=v$. Second, the precedence relation of a proper structure must be irreflexive if the structure is presentist or expansionist. Third, the condition that $\mathscr{T}$ has at least two members in the definition of 'proper' is there to capture the view that there is (a minimal amount of) temporal passage. Without this condition, there would be proper structures that are both presentist and expansionist, while with this condition there can be none such.

Let $\mathbb{L}$ be any temporal language. We define a model for $\mathbb{L}$ as a tuple $\langle\mathscr{T},\langle, D, I\rangle$, where $\langle\mathscr{T},<, D\rangle$ is a structure and $I$ (interpretation) a function taking each constant of the language into an element of $\mathscr{D}=\bigcup_{u \in \mathscr{T}} D(u)$, and each $k$-place predicate of the language and each time into a set of $k$-tuples of elements of $\mathscr{D}$, with the usual constraint regarding the identity predicate:

- For all $t \in \mathscr{T},\left\langle o, o^{*}\right\rangle \in I(=, t)$ iff $o=o^{*}$. 
The model is called proper if the underlying structure is proper, and if in addition $I$ satisfies the following condition regarding $\mathrm{T}$ :

- For all $t \in \mathscr{T},\langle o\rangle \in I(\mathrm{~T}, t)$ iff $o \in \mathscr{T}$.

We say that a proper model for $\mathbb{L}$ is presentist / expansionist iff the underlying structure is presentist / expansionist.

The truth-conditions for the $\mathbb{L}$-formulas in model $M=\langle\mathscr{T},\langle, D, I\rangle$ at a time $t$ relative to a variable-assignment $r$ for $\mathbb{L}$ are specified as follows $\left(I_{r}(m)\right.$ is $I(m)$ if $m$ is a constant, and $r(m)$ if $m$ is a variable):

- $t \models_{r}^{M} \mathrm{~F} m_{1} \ldots m_{k}$ iff $\left\langle I_{r}\left(m_{1}\right), \ldots, I_{r}\left(m_{k}\right)\right\rangle \in I(\mathrm{~F}, t)$ for any $k$-place predicate $\mathrm{F}$.

- $t \models_{r}^{M} \neg \phi$ iff $t \not \nvdash_{r}^{M} \phi$.

- $t \models_{r}^{M} \phi \vee \psi$ iff $t \models_{r}^{M} \phi$ or $t \models_{r}^{M} \psi$.

- $t \models_{r}^{M} \exists x \phi$ iff $t \models_{r^{\prime}}^{M} \phi$ for some variable-assignment $r^{\prime}$ differing from $r$ at most on $x$ such that $r^{\prime}(x) \in D(t)$.

- $t \models_{r}^{M} H \phi$ iff $t^{\prime} \vDash_{r}^{M} \phi$ for all $t^{\prime} \in \mathscr{T}$ such that $t^{\prime}<t$.

- $t \models_{r}^{M} G \phi$ iff $t^{\prime} \models_{r}^{M} \phi$ for all $t^{\prime} \in \mathscr{T}$ such that $t<t^{\prime}$.

We then have:

- $t \models_{r}^{M} \forall x \phi$ iff $t \vDash_{r^{\prime}} \phi$ for all variable-assignments $r^{\prime}$ differing from $r$ at most on $x$ such that $r^{\prime}(x) \in D(t)$.

- $t \models_{r}^{M} P \phi$ iff $t^{\prime} \vDash_{r} \phi$ for some $t^{\prime} \in \mathscr{T}$ such that $t^{\prime}<t$.

- $t \vDash_{r}^{M} F \phi$ iff $t^{\prime} \vDash_{r} \phi$ for some $t^{\prime} \in \mathscr{T}$ such that $t<t^{\prime}$.

- $t \vDash_{r}^{r} S \phi$ iff $t^{\prime} \vDash_{r} \phi$ for some $t^{\prime} \in \mathscr{T}$.

- $t \models_{r}^{M} A \phi$ iff $t^{\prime} \vDash_{r} \phi$ for all $t^{\prime} \in \mathscr{T}$.

- $t \models_{r}^{M} \mathrm{E} m$ iff $I_{r}(m) \in D(t)$.

Where $\phi$ is an $\mathbb{L}$-formula and $M$ a model for $\mathbb{L}, \phi$ is said to $\mathbb{L}$-hold in $M$ iff $\phi$ is true at all times of $M$ relative to all variable-assignments for $\mathbb{L}$.

The models for temporal languages as they have been defined here are nothing but standard variable domain models for first-order quantified Priorean languages with identity, and the semantic clauses put forward are also completely standard. What is non-standard about the proposed semantics is the focus on proper models, more precisely on models in which each point of evaluation belongs to its own domain, and which assign to a distinguished predicate ( $T$, in our case) an extension whose members are points of evaluation.

The reader will have noticed that nothing has been said about the other distinguished predicate of temporal languages, namely the temporal location predicate $\mathrm{L}$. There are indeed a number of different ways of characterising the notion of temporal location that are available in the present context, and on our view none of these characterisations is more suited to subserve one version of temporaryism rather than the other. Rather than making a particular decision on this issue right from the start, or already working with a full range of different options, we prefer, for the sake of perspicuity, to postpone the treatment of temporal location (see section 8).

\section{Digression: presentness, precedence and truth}

Consider a temporal language with a monadic predicate PRES intended to express the property of being a present time, a binary predicate $<$ for precedence between times, enriched with a truth operator @ such that for $m$ a term and $\phi$ a formula, $@_{m} \phi$ is a well-formed formula, intended to express the claim that it is true at 
time $m$ that $\phi$. In order to properly interpret this language, the previous semantics should be extended by adding the following truth-conditions relative to any model $M=\langle\mathscr{T},<, D, I\rangle$ and variable-assignment $r$ :

$$
\begin{array}{ll}
\text { - } t \models_{r}^{M} \text { PRES } m \text { iff } I_{r}(m)=t . \\
\text { - } t \models_{r}^{M} m<n \text { iff } I_{r}(m), I_{r}(n) \in \mathscr{T} \text { and } I_{r}(m)<I_{r}(n) . \\
\text { - } t \models_{r}^{M} @_{m} \phi \text { iff } I_{r}(m) \in \mathscr{T} \text { and } I_{r}(m) \models_{r}^{M} \phi .
\end{array}
$$

Interestingly, relative to presentist models as well as relative to expansionist models, the special predicates and the special operator are definable (in the logical sense) using resources which belong to all temporal languages. Thus, relative to expansionist models,

- PRES $m$ is definable as $\mathrm{T} m \wedge \mathrm{E} m \wedge H \neg \mathrm{E} m$,

- $m<n$ as $\mathrm{T} m \wedge \mathrm{T} n \wedge A(\mathrm{E} n \supset P \mathrm{E} m)$, and

- @ $m \phi$ as $\mathrm{T} m \wedge A(\mathrm{E} m \wedge H \neg \mathrm{E} m \supset \phi)$.

The very same definability facts hold relative to the presentist models, although in this case we also have that

- PRES $m$ is definable as $\mathrm{T} m \wedge \mathrm{E} m$, and

- $@_{m} \phi$ as $\mathrm{T} m \wedge A(\mathrm{E} m \supset \phi)$.

Of course, these points concern logical definability; presentists and expansionists are in principle free to accept the corresponding equivalences without regarding them as reductive (nominal or real) definitions. Instead, they may take some or all of the three notions as irreducible. In the present framework, the right thing for them to do would then be to consider languages with the relevant predicate(s) or operator together with the corresponding semantics.

As we stressed in the introduction to this paper, we believe that there might well be no metaphysically substantive notion of presentness, and that accordingly both presentism and expansionism should be characterised without appeal to such a notion. Our view on the notions of precedence between times and truth-at-a-time is different: we do believe that these notions are metaphysically significant. Accordingly, we are in principle ready to accept formulations of presentist and expansionist logics in languages containing both $<$ and $@ .^{5}$ Our view, though, is that the expressive resources introduced in section 2 suffice to capture both the core of presentism and the core of expansionism.

\section{The systems}

We here define three Hilbert-style systems (relative to any given temporal language): the neutral system, the presentist system Pres, and the expansionist system Exp.

The neutral system is defined by means of the following three sets of postulates:

\section{AXIOMS AND RULE FROM THE PROPOSITIONAL CALCULUS}

Axioms: All instances of classical tautologies.

Rule: Modus Ponens.

\section{AXIOMS AND RULES FOR THE PRIOREAN OPERATORS}

A1: $\phi \supset H F \phi$

A2: $\phi \supset G P \phi$

A3: $H(\phi \supset \psi) \supset(H \phi \supset H \psi)$ 

A4: $G(\phi \supset \psi) \supset(G \phi \supset G \psi)$
A5: $F P \phi \supset(P \phi \vee \phi \vee F \phi)$
A6: $P F \phi \supset(P \phi \vee \phi \vee F \phi)$
A7: $P P \phi \supset P \phi$
R1: $\phi / H \phi$
R2: $\phi / G \phi$

REMARK: A1-A4 plus R1 and R2, in addition to the classical postulates mentioned above, define minimal tense logic. The remaining axioms correspond to the following conditions on frames in the standard Kripke semantics: A5 corresponds to the condition that the temporal ordering is linear in the past, $\mathrm{A} 6$ to the condition that the temporal ordering is linear in the future, A7 to the condition that the temporal ordering is transitive (given the rest of the postulates, we could have laid down $F F \phi \supset F \phi$ instead of A7).

\section{AXIOMS AND RULE FOR QUANTIFICATION AND IDENTITY}
A8: $\forall x(\phi \supset \psi) \supset(\forall x \phi \supset \forall x \psi)$
A9: $\phi \supset \forall x \phi$
A10: $\forall x \mathrm{E} x$
A11: $\forall x \phi \supset(\mathrm{E} m \supset \phi[m / x])$
A12: $x=x$
A13: $x=y \supset(\phi \supset \phi[y / / x])$
A14: $S \mathrm{E} m$
R3: $\phi / \forall x \phi$

In $\mathrm{A} 9, x$ is supposed not to be free in $\phi$. In A11, $\phi[m / x]$ is the result of freely replacing each free occurrence of $x$ in $\phi$ by $m$. In A13, $\phi[y / / x]$ is the result of freely replacing zero or more free occurrences of $x$ in $\phi$ by $y$.

REMARK: This is a standard set of postulates for free logic in quantified temporal logic.

The neutral system is characterised by the models introduced in the previous section, i.e. a formula is a theorem of the system iff it holds in all models. We will come back to this in section 6.2.

The presentist system and the expansionist system both result from the neutral system by adding extra axioms. They share the following axioms for $\mathrm{T}$ :

$$
\begin{aligned}
& \text { AT1: } \mathrm{T} x \supset A T x \\
& \text { AT2: } S \exists x(T x \wedge S \exists y(T y \wedge x \neq y)
\end{aligned}
$$

Pres is defined by adding the following axioms:

$$
\begin{aligned}
& \text { AP1: } \exists x \mathrm{~T} x \\
& \text { AP2: } \forall x \forall y(\mathrm{~T} x \wedge \mathrm{T} y \supset x=y) \\
& \text { AP3: } \forall x(\mathrm{~T} x \supset H \neg \mathrm{E} x)
\end{aligned}
$$

In English: there's a unique time, and that time never existed before. Exp is defined by adding the following axioms instead:
AE1: $\exists x(\mathrm{~T} x \wedge H \neg \mathrm{E} x)$
AE2: $\forall x \forall y(\mathrm{~T} x \wedge \mathrm{T} y \wedge H \neg \mathrm{E} x \wedge H \neg \mathrm{E} y \supset x=y)$
AE3: $\forall x(\mathrm{~T} x \supset H \neg \mathrm{E} x \vee P(\mathrm{E} x \wedge H \neg \mathrm{E} x))$
AE4: $\forall x G \mathrm{E} x$ 
In English: there's a unique time that never existed before, every time either never existed before or sometimes in the past existed without ever existing before, and whatever exists will never fail to exist.

It is possible to replace AE1-AE3 by an equivalent but somewhat less cumbersome set of axioms formulated using the predicate $\mathrm{N}$ (read: 'is new'), where $\mathrm{N} m$ is defined as $\mathrm{E} m \wedge H \neg \mathrm{E} m$ :

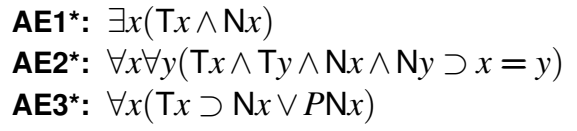

In English: there's a unique new time, and every time either is or was new. Likewise, AP3 could be replaced by

AP3 $^{*}: \forall x(T x \supset \mathrm{N} x)$

\section{Soundness and completeness}

Let $\mathbb{L}$ be a temporal language, held fixed throughout this section, in which we assume that Pres and Exp are formulated. The aim is to show that an $\mathbb{L}$-formula is a theorem of Pres iff it $\mathbb{L}$-holds in all presentist models for $\mathbb{L}$, and that an $\mathbb{L}$-formula is a theorem of Exp iff it $\mathbb{L}$-holds in all expansionist models for $\mathbb{L}$. We establish the "only if" direction of these two claims in section 6.1, and the "if" direction in section 6.3 .

\subsection{Soundness for Pres and Exp}

Theorem 6.1 (Soundness) $\quad$ (1) An $\mathbb{L}$-formula is a theorem of Pres only if it $\mathbb{L}$ holds in all presentist models for $\mathbb{L}$. (2) An $\mathbb{L}$-formula is a theorem of Exp only if it $\mathbb{L}$-holds in all expansionist models for $\mathbb{L}$.

Proof Let us keep reference to $\mathbb{L}$ implicit for the sake of readability. It is routine to verify that all the axioms of the neutral system hold in every model, and that each of Modus Ponens, R1, R2 and R3 preserves the property of holding in a model. This establishes that the neutral system is sound with respect to the class of all models, i.e. that a formula is a theorem of the system only if it holds in all models. What remains to be done in order to establish (1) and (2) is to verify that (a) AT1 and AT2 hold in every proper model, (b) AP1-AP3 hold in every presentist model, and (c) AE1-AE4 hold in every expansionist model. (a) is obvious given how proper models are required to interpret $\mathrm{T}$. Let us move to $(\mathrm{b})$ and $(\mathrm{c})$ :

(b) Let $M=\langle\mathscr{T},<, D, I\rangle$ be an arbitrary presentist model, $t$ a time of the model and $r$ a variable-assignment.

AP1. $t \models_{r}^{M} \exists x \mathrm{~T} x$ because $M$ is a proper model.

AP2. $t \models_{r}^{M} \forall x \forall y(\mathrm{~T} x \wedge \mathrm{T} y \supset x=y)$ because given that $\langle\mathscr{T},<, D\rangle$ is a presentist structure, for all $u, v \in \mathscr{T}, u \in D(v) \Rightarrow u=v$ (as we emphasised right after defining the various kinds of structures in section 3$)$.

AP3. Suppose, for reductio, that there is a variable-assignment $r^{\prime}$ with $r^{\prime}(x) \in D(t)$ and $t \not \nvdash_{r^{\prime}}^{M} \mathrm{~T} x \supset H \neg \mathrm{E} x$. Then $r^{\prime}(x) \in \mathscr{T}$ and $r^{\prime}(x) \in D\left(t^{\prime}\right)$ for some $t^{\prime}$ such that $t^{\prime}<t$. But then $r^{\prime}(x)=t$ (see the previous point), and so we have both $t \in D\left(t^{\prime}\right)$ and $t^{\prime}<t$. But this is impossible, due to condition (c2) in the definition of a presentist structure.

(c) Let $M=\langle\mathscr{T},\langle, D, I\rangle$ be an arbitrary expansionist model, $t$ a time of the model and $r$ a variable-assignment. 
AE1. Let $r^{\prime}$ be the variable-assignment such that $r^{\prime}(x)=t$ that agrees with $r$ on all variables distinct from $x$. Then by condition (c2) in the definition of an expansionist structure, there is no $t^{\prime}$ such that $t^{\prime}<t$ and $r^{\prime}(x) \in D\left(t^{\prime}\right)$. It follows that $t \models_{r}^{M} \exists x(\mathrm{~T} x \wedge H \neg \mathrm{E} x)$.

AE2. Let $r^{\prime}$ be a variable-assignment such that $r^{\prime}(x), r^{\prime}(y) \in D(t)$ and $t \models_{r^{\prime}}^{M} \mathrm{~T} x \wedge$ $\mathrm{T} y \wedge H \neg \mathrm{E} x \wedge H \neg \mathrm{E} y$. Then $r^{\prime}(x) \in \mathscr{T}$, and for no $t^{\prime}<t$ is it the case that $r^{\prime}(x) \in D\left(t^{\prime}\right)$. Since $M$ 's structure is proper, we have in particular that it is not the case that $r^{\prime}(x)<t$. Now since both $r^{\prime}(x) \in \mathscr{T}$ and $r^{\prime}(x) \in D(t)$, we also have, thanks to condition (c2) in the definition of an expansionist structure, that it is not the case that $t<r^{\prime}(x)$. Given the totality of $<$, we must then have $r^{\prime}(x)=t$. A strictly similar argument establishes that $r^{\prime}(y)=t$. It follows that $t \models_{r^{\prime}}^{M} x=y$. Hence, $t \models_{r}^{M} \forall x \forall y(\mathrm{~T} x \wedge \mathrm{T} y \wedge H \neg \mathrm{E} x \wedge H \neg \mathrm{E} y \supset x=y)$.

AE3. Let $r^{\prime}$ be a variable-assignment such that $r^{\prime}(x) \in D(t)$ and $t \models_{r^{\prime}}^{M} \mathrm{~T} x$. Then $r^{\prime}(x) \in \mathscr{T}$. By the totality of $<$ and condition (c2) in the definition of an expansionist structure, we must then have $r^{\prime}(x)=t$ or $r^{\prime}(x)<t$. In the first case, condition (ii) guarantees that we have $t \models_{r^{\prime}}^{M} H \neg \mathrm{E} x$. In the second case, the same condition and the fact that $M$ is proper jointly guarantee that we have $t \models_{r^{\prime}}^{M} P(\mathrm{E} x \wedge H \neg \mathrm{E} x)$. Hence, we have $t \models_{r}^{M} \forall x(\mathrm{~T} x \supset H \neg \mathrm{E} x \vee P(\mathrm{E} x \wedge H \neg \mathrm{E} x))$.

AE4. Let $r^{\prime}$ be a variable-assignment such that $r^{\prime}(x) \in D(t)$, and let $t^{\prime}$ be such that $t<t^{\prime}$. By condition (exp) in the definition of an expansionist system, then, $r^{\prime}(x) \in D\left(t^{\prime}\right)$. We thus have $t \models_{r^{\prime}}^{M} G \mathrm{E} x$. It follows that $t \models_{r}^{M} \forall x G \mathrm{E} x$.

6.2 The standard canonical construction For the completeness proofs, we piggyback on a standard canonical construction and provide a new construction on its basis. ${ }^{6}$ We here run through the standard construction.

Where $\mathbf{S}^{*}$ is a Hilbert-style system formulated in a language $\mathbb{L}^{*}$ that is an extension of a standard first-order language, say that a set of $\mathbb{L}^{*}$-formulas $\Gamma$ is:

- $\mathbf{S}^{*}$-consistent iff for no $\phi_{1}, \ldots, \phi_{n} \in \Gamma$ is it the case that $\neg\left(\phi_{1} \wedge \ldots \wedge \phi_{n}\right)$ a theorem of $\mathbf{S}^{*}$.

- $\mathbb{L}^{*}$-maximal iff for any $\mathbb{L}^{*}$-formula $\phi, \phi \in \Gamma$ or $\neg \phi \in \Gamma$.

- $\mathbb{L}^{*}-\exists$-saturated iff if $\exists x \delta \in \Gamma$, then for some $\mathbb{L}^{*}$-variable $y$, Ey $y \delta[y / x] \in \Gamma$.

- $\mathbb{L}^{*}-\diamond \exists$-saturated iff for all $n \geq 1$, all $\mathbb{L}^{*}$-formulas $\delta_{1}, \ldots, \delta_{n}$ and $\delta$, and all $\diamond_{1}, \ldots, \diamond_{n}$ in $\{P, F\}$, if $\nabla_{n}\left(\delta_{n} \wedge \nabla_{n-1}\left(\ldots \diamond_{1}\left(\delta_{1} \wedge \exists x \delta\right) \ldots\right)\right) \in \Gamma$, then for some $L$-variable $y, \diamond_{n}\left(\delta_{n} \wedge \nabla_{n-1}\left(\ldots \nabla_{1}\left(\delta_{1} \wedge \mathrm{E} y \wedge \delta[y / x]\right) \ldots\right)\right) \in \Gamma$.

- $\mathbf{S}^{*}$-nice iff it has the previous four properties.

Let $\mathbb{L}^{+}$be a temporal language obtained from $\mathbb{L}$ by adding a countably infinite set of new variables. Up until the formulation of proposition 6.8 below, $\mathbf{S}$ will be any given extension of the neutral system formulated in $\mathbb{L}$, and $\mathbf{S}^{+}$will be the same extension but formulated in $\mathbb{L}^{+}$.

Proposition 6.2 (Lindenbaum Lemma) Every $\mathbf{S}$-consistent set of $\mathbb{L}$-formulas can be extended to an $\mathbf{S}^{+}$-nice set of $\mathbb{L}^{+}$-formulas.

Proof Let $\Sigma$ be an $\mathbf{S}$-consistent set of $\mathbb{L}$-formulas. Suppose given an enumeration of all the $\mathbb{L}^{+}$-formulas, and an enumeration of all the $\mathbb{L}^{+}$-variables. Define a series $\Sigma_{0}, \Sigma_{1}, \ldots$ of sets of $\mathbb{L}^{+}$-formulas as follows:

1. $\Sigma_{0}=\Sigma$.

2. If $\phi_{k+1}$ is the $(k+1)^{\text {th }} \mathbb{L}^{+}$-formula of our enumeration $(k \geq 0), \Sigma_{k+1}$ is defined according to the following conditions: 
(a) If $\Sigma_{k} \cup\left\{\phi_{k+1}\right\}$ is not $\mathbf{S}^{+}$-consistent, then $\Sigma_{k+1}=\Sigma_{k}$.

(b) If $\Sigma_{k} \cup\left\{\phi_{k+1}\right\}$ is $\mathbf{S}^{+}$-consistent and $\phi_{k+1}$ is neither of the form $\exists x \boldsymbol{\delta}$ nor of the form $\nabla_{n}\left(\delta_{n} \wedge \nabla_{n-1}\left(\ldots \nabla_{1}\left(\delta_{1} \wedge \exists x \delta\right) \ldots\right)\right)$, then $\Sigma_{k+1}=\Sigma_{k} \cup\left\{\phi_{k+1}\right\}$.

(c) If $\Sigma_{k} \cup\left\{\phi_{k+1}\right\}$ is $\mathbf{S}^{+}$-consistent and $\phi_{k+1}$ is of the form $\exists x \delta$, then $\Sigma_{k+1}=\Sigma_{k} \cup\left\{\phi_{k+1}\right\} \cup\{\mathrm{E} y \wedge \delta[y / x]\}$, where $y$ is the first $\mathbb{L}^{+}$-variable not to appear in $\Sigma_{k}$ or in $\phi_{k+1}$ (there are such variables, since $\mathbb{L}^{+}$has infinitely more variables than $\mathbb{L}$ ).

(d) If $\Sigma_{k} \cup\left\{\phi_{k+1}\right\}$ is $\mathbf{S}^{+}$-consistent and $\phi_{k+1}$ is of the form $\nabla_{n}\left(\delta_{n} \wedge \nabla_{n-1}(\ldots\right.$ $\left.\left.\diamond_{1}\left(\delta_{1} \wedge \exists x \delta\right) \ldots\right)\right)$, then $\Sigma_{k+1}=\Sigma_{k} \cup\left\{\phi_{k+1}\right\} \cup\left\{\nabla_{n}\left(\delta_{n} \wedge \diamond_{n-1}\left(\ldots \diamond_{1}\left(\delta_{1} \wedge\right.\right.\right.\right.$ $\mathrm{E} y \wedge \delta[y / x]) \ldots))\}$, where $y$ is the first $\mathbb{L}^{+}$-variable not to appear in $\Sigma_{k}$ or in $\phi_{k+1}$ (again, there are such variables).

Define $\Sigma^{+}=\bigcup_{k \geq 0} S_{k}$. By construction, $\Sigma^{+}$is $\mathbb{L}^{+}$-maximal, $\mathbb{L}^{+}-\exists$-saturated and $\mathbb{L}^{+}$$\diamond \exists$-saturated. Given that $\Sigma_{0}$ is $\mathbf{S}$-consistent, it is $\mathbf{S}^{+}$-consistent. Thus if we can establish that construction steps (c) and (d) preserve $\mathbf{S}^{+}$-consistency, then we will have established that all the $\Sigma_{k}$ s, and hence $\Sigma^{+}$is itself, are $\mathbf{S}^{+}$-consistent, and the proposition will be proved.

That step (c) preserves $\mathbf{S}^{+}$-consistency. Assume that $\Sigma_{k} \cup\{\exists x \boldsymbol{\delta}\} \cup\{\mathrm{E} y \wedge \boldsymbol{\delta}[y / x]\}$ is not $\mathbf{S}^{+}$-consistent. Then for some $\psi_{1}, \ldots, \psi_{l}$ in $\Sigma_{k} \cup\{\exists x \delta\},\left(\psi_{1} \wedge \ldots \wedge \psi_{l}\right) \supset($ Ey $\supset$ $\neg \delta[y / x])$ is a theorem of $\mathbf{S}^{+}$. Then by R3 and A9, $\left(\psi_{1} \wedge \ldots \wedge \psi_{l}\right) \supset \forall y(\mathrm{E} y \supset \neg \delta[y / x])$ is a theorem of $\mathbf{S}^{+}$, and hence by A8 and A10, so is $\left(\psi_{1} \wedge \ldots \wedge \psi_{l}\right) \supset \forall y \neg \delta[y / x]$. It follows that $\Sigma_{k} \cup\{\exists x \delta\}$ is not $\mathbf{S}^{+}$-consistent.

That step (d) preserves $\mathbf{S}^{+}$-consistency. The proof will make crucial use of the following fact:

FACT. $\alpha \supset H \beta$ is a theorem of $\mathbf{S}^{+}$iff $F \alpha \supset \beta$ is, and likewise $\alpha \supset G \beta$ is a theorem of $\mathbf{S}^{+}$iff $P \alpha \supset \beta$ is.

(Only postulates of minimal tense logic are needed to establish this.)

Assume that $\Sigma_{k} \cup\left\{\nabla_{n}\left(\delta_{n} \wedge \nabla_{n-1}\left(\ldots \diamond_{1}\left(\delta_{1} \wedge \exists x \delta\right) \ldots\right)\right)\right\} \cup\left\{\nabla_{n}\left(\delta_{n} \wedge \nabla_{n-1}\left(\ldots \diamond_{1}\left(\delta_{1} \wedge\right.\right.\right.\right.$ E $y \wedge \delta[y / x]) \ldots))\}$ is not $\mathbf{S}^{+}$-consistent. Then for some $\psi_{1}, \ldots, \psi_{l}$ in $\Sigma_{k} \cup\left\{\nabla_{n}\left(\delta_{n} \wedge\right.\right.$ $\left.\left.\diamond_{n-1}\left(\ldots \diamond_{1}\left(\delta_{1} \wedge \exists x \delta\right) \ldots\right)\right)\right\}$,

- $\left(\psi_{1} \wedge \ldots \wedge \psi_{l}\right) \supset \square_{n}\left(\delta_{n} \supset \square_{n-1}\left(\ldots \square_{1}\left(\delta_{1} \supset(\mathrm{E} y \supset \neg \delta[y / x])\right) \ldots\right)\right)$

is a theorem of $\mathbf{S}^{+}$, where $\square_{i}=H$ if $\diamond_{i}=P$, and $\square_{i}=G$ if $\diamond_{i}=F$. Thanks to FACT,

- $\left(\wedge_{1}\left(\ldots\left(\diamond_{n-1}\left(\diamond_{n}\left(\psi_{1} \wedge \ldots \wedge \psi_{l}\right) \wedge \delta_{n}\right)\right) \ldots\right) \wedge \delta_{1}\right) \supset(\mathrm{E} y \supset \neg \delta[y / x])$

is then also a theorem of $\mathbf{S}^{+}$, where ${ }_{i}=F$ if $\square_{i}=H$, and $\boldsymbol{}_{i}=P$ if $\square_{i}=G$. Reasoning as in case (c), we infer that

- $\left(\wedge_{1}\left(\ldots\left(\diamond_{n-1}\left(\diamond_{n}\left(\psi_{1} \wedge \ldots \wedge \psi_{l}\right) \wedge \delta_{n}\right)\right) \ldots\right) \wedge \delta_{1}\right) \supset \forall y \neg \delta[y / x]$

is a theorem of $\mathbf{S}^{+}$. Using FACT again, we conclude that

- $\left(\psi_{1} \wedge \ldots \wedge \psi_{l}\right) \supset \square_{n}\left(\delta_{n} \supset \square_{n-1}\left(\ldots \square_{1}\left(\delta_{1} \supset \forall y \neg \delta[y / x]\right) \ldots\right)\right)$

is also a theorem of $\mathbf{S}^{+}$. It follows that $\Sigma_{k} \cup\left\{\nabla_{n}\left(\delta_{n} \wedge \nabla_{n-1}\left(\ldots \diamond_{1}\left(\delta_{1} \wedge \exists x \delta\right) \ldots\right)\right)\right\}$ is not $\mathbf{S}^{+}$-consistent.

Define the binary relation $<\mathbf{S}^{+}$between $\mathbf{S}^{+}$-nice sets of $\mathbb{L}^{+}$-formulas as follows: $\Gamma<\mathbf{S}^{+} \Delta$ iff $\phi \in \Gamma$ whenever $H \phi \in \Delta$. Note that thanks to A1 and A2, we then have: $\Gamma<\mathbf{S}^{+} \Delta$ iff $\phi \in \Delta$ whenever $H \phi \in \Gamma$. The proof of the following proposition is routine: 


\section{Proposition 6.3}

1. $<\mathbf{S}^{+}$is transitive. (Use A7 for the proof.)

2. $<\mathbf{S}^{+}$is linear towards the past, i.e. for all $\Delta, \Delta^{*}$ and $\Gamma$, if both $\Delta<\mathbf{S}^{+} \Gamma$ and $\Delta^{*}<\mathbf{S}^{+} \Gamma$, then either $\Delta=\Delta^{*}$, or $\Delta<\mathbf{S}^{+} \Delta^{*}$, or $\Delta^{*}<\mathbf{S}^{+} \Delta$. (Use A5 for the proof.)

3. $<^{+}$is linear towards the future, i.e. for all $\Delta, \Delta^{*}$ and $\Gamma$, if both $\Gamma<^{\mathbf{S}^{+}} \Delta$ and $\Gamma<\mathbf{S}^{+} \Delta^{*}$, then either $\Delta=\Delta^{*}$, or $\Delta<^{\mathbf{S}^{+}} \Delta^{*}$, or $\Delta^{*}<^{\mathbf{S}^{+}} \Delta$. (Use A6 for the proof.)

Proposition 6.4 For all $\mathbf{S}^{+}$-nice sets of $\mathbb{L}^{+}$-formulas $\Delta$ and all $\mathbb{L}^{+}$-formulas $\phi$ :

1. $H \phi \in \Delta$ iff for all $\mathbf{S}^{+}$-nice set of $\mathbb{L}^{+}$-formulas $\Gamma$ such that $\Gamma<^{\mathbf{S}^{+}} \Delta, \phi \in \Gamma$.

2. $G \phi \in \Delta$ iff for all $\mathbf{S}^{+}$-nice set of $\mathbb{L}^{+}$-formulas $\Gamma$ such that $\Delta<\mathbf{S}^{+} \Gamma, \phi \in \Gamma$.

Proof We only go through the proof of the first claim, the proof of the second claim being almost identical. The left-to-right direction is immediate. For the other direction, we first establish the following proposition:

- Let $\Delta$ be an $\mathbf{S}^{+}$-nice sets of $\mathbb{L}^{+}$-formulas such that $H \phi \notin \Delta$. Then there is a set of $\mathbb{L}^{+}$-formulas $\Gamma$ that is $\mathbf{S}^{+}$-consistent, $\mathbb{L}^{+}-\exists$-saturated and $\mathbb{L}^{+}-\diamond \exists$ saturated, and such that $\{\neg \phi\} \cup\{\psi: H \psi \in \Delta\} \subseteq \Gamma$.

The proposition yields the desired result. For let $\Gamma^{*}$ be an $\mathbb{L}^{+}$-maximal, $\mathbf{S}^{+}$consistent extension of $\Gamma$. By construction, $\Gamma^{*}$ is both $\mathbb{L}^{+}-\exists$-saturated and $\mathbb{L}^{+}-\diamond \exists$ saturated, and hence it is $\mathbf{S}^{+}$-nice. Since $\Gamma^{*}$ extends $\Gamma$, we have $\Gamma^{*}<\mathbf{S}^{+} \Delta$ and $\phi \notin \Gamma^{*}$.

Now to the proof of the proposition. Let $\Delta$ be as stated, and let $\Omega$ be $\{\psi: H \psi \in \Delta\}$. Enumerate all the $\mathbb{L}^{+}$-formulas of type $\exists x \delta$ or $\nabla_{n}\left(\delta_{n} \wedge \nabla_{n-1}\left(\ldots \diamond_{1}\left(\delta_{1} \wedge \exists x \delta\right) \ldots\right)\right)$, labelling them $\phi_{1}, \phi_{2}, \ldots$, and also enumerate all the $\mathbb{L}^{+}$-variables. We build a series of sets of $\mathbb{L}^{+}$-formulas $\Gamma_{0}, \Gamma_{1}, \ldots$ with:

1. $\Gamma_{0}=\Omega \cup\{\neg \phi\}$.

2. If $\Gamma_{n} \cup\left\{\phi_{n+1}\right\}$ is $\mathbf{S}^{+}$-inconsistent, then $\Gamma_{n+1}=\Gamma_{n}$.

3. If $\Gamma_{n} \cup\left\{\phi_{n+1}\right\}$ is $\mathbf{S}^{+}$-consistent, then:

3.1 For the case in which $\phi_{n+1}$ is $\exists x \delta$ :

$\Gamma_{n+1}=\Gamma_{n} \cup\{\exists x \delta\} \cup\{\mathrm{E} y \wedge \delta[y / x]\}$, where $y$ is the first $\mathbb{L}^{+}$-variable such that $\Gamma_{n} \cup\{\exists x \delta\} \cup\{\mathrm{E} y \wedge \delta[y / x]\}$ is $\mathbf{S}^{+}$-consistent.

3.2 For the case in which $\phi_{n+1}$ is $\nabla_{n}\left(\delta_{n-1} \wedge \nabla_{n-1}\left(\ldots \nabla_{1}\left(\delta_{1} \wedge \exists x \delta\right) \ldots\right)\right)$ :

$\Gamma_{n+1}=\Gamma_{n} \cup\left\{\diamond_{n}\left(\delta_{n-1} \wedge \nabla_{n-1}\left(\ldots \diamond_{1}\left(\delta_{1} \wedge \exists x \delta\right) \ldots\right)\right)\right\} \cup\left\{\nabla_{n}\left(\delta_{n-1} \wedge \nabla_{n-1}\right.\right.$ $\left.\left.\left(\ldots \nabla_{1}\left(\delta_{1} \wedge E y \wedge \delta[y / x]\right) \ldots\right)\right)\right\}$, where $y$ is the first $\mathbb{L}^{+}$-variable such that $\Gamma_{n} \cup\left\{\nabla_{n}\left(\delta_{n-1} \wedge \diamond_{n-1}\left(\ldots \diamond_{1}\left(\delta_{1} \wedge \exists x \delta\right) \ldots\right)\right)\right\} \cup\left\{\nabla_{n}\left(\delta_{n-1} \wedge \diamond_{n-1}\left(\ldots \diamond_{1}\right.\right.\right.$ $\left.\left.\left.\left(\delta_{1} \wedge \mathrm{E} y \wedge \delta[y / x]\right) \ldots\right)\right)\right\}$ is $\mathbf{S}^{+}$-consistent.

We need to show that in each of the cases 3.1 and 3.2, there is an $\mathbb{L}^{+}$-variable $y$ satisfying the corresponding condition. Given that $\Gamma_{0}$ is $\mathbf{S}^{+}$-consistent, once this is done, the proposition will be established: the union of all the $\Gamma_{n} \mathrm{~s}$ has all the properties required.

Suppose, then, that $\Gamma_{n} \cup\left\{\phi_{n+1}\right\}$ is $\mathbf{S}^{+}$-consistent, and let us show that in each of the cases 3.1 and 3.2 , there is a variable satisfying the relevant condition. 
Consider first case 3.2. Let us abbreviate $\nabla_{n}\left(\delta_{n-1} \wedge \nabla_{n-1}\left(\ldots \diamond_{1}\left(\delta_{1} \wedge X\right) \ldots\right)\right)$ to $\Xi(X)$. Assume for reductio that there is no $\mathbb{L}^{+}$-variable $y$ satisfying the condition mentioned, i.e. that for every $\mathbb{L}^{+}$-variable $y, \Gamma_{n} \cup\{\Xi(\exists x \delta)\} \cup\{\Xi(\mathrm{E} y \wedge \delta[y / x])\}$ is not $\mathbf{S}^{+}$-consistent. Let $\Theta$ be $\Gamma_{n}-\Omega$. $\Theta$ is thus finite. Let $y$ be any arbitrary $\mathbb{L}^{+}$-variable. Then for some $\left\{\omega_{1}, \ldots, \omega_{m}\right\} \subseteq \Omega,\left(\omega_{1} \wedge \ldots \wedge \omega_{m}\right) \supset(\wedge \Theta \wedge \Xi(\exists x \delta) \supset \neg \Xi(E y \wedge \delta[y / x]))$ is a theorem of $\mathbf{S}^{+}$. (In case $\Theta$ is empty, let $\wedge \Theta$ be any tautology without free variables.) We infer that $\left(H \omega_{1} \wedge \ldots \wedge H \omega_{m}\right) \supset H(\wedge \Theta \wedge \Xi(\exists x \delta) \supset \neg \Xi(E y \wedge \delta[y / x]))$ is also a theorem of $\mathbf{S}^{+}$. Given that $\Delta$ is both $\mathbb{L}^{+}$-maximal and $\mathbf{S}^{+}$-consistent, it follows that $H(\wedge \Theta \wedge \Xi(\exists x \delta) \supset \neg \Xi(E y \wedge \delta[y / x])) \in \Delta$.

We then want to prove that for some $\mathbb{L}^{+}$-variable $z, H(\wedge \Theta \wedge \Xi(\exists x \delta) \supset \neg \Xi(\exists z(\mathrm{E} z \wedge$ $\delta[z / x]))) \in \Delta$. Take any $\mathbb{L}^{+}$-variable $z$ not in $\delta$. Assume for reductio that $H(\wedge \Theta \wedge \Xi(\exists x \delta) \supset \neg \Xi(\exists z(\mathrm{E} z \wedge \delta[z / x]))) \notin \Delta$. Then $P(\wedge \Theta \wedge \Xi(\exists x \delta) \wedge \Xi(\exists z(\mathrm{E} z \wedge$ $\delta[z / x]))) \in \Delta$. Since $\Delta$ is $\mathbb{L}^{+}-\diamond \exists$-saturated, we then have $P(\wedge \Theta \wedge \Xi(\exists x \delta) \wedge \Xi(\mathrm{E} t \wedge$ $\delta[t / x])) \in \Delta$ for some $\mathbb{L}^{+}$-variable $t$. By the fact established in the previous paragraph, it follows that $P(\Xi(\mathrm{E} t \wedge \delta[t / x]) \wedge \neg \Xi(\mathrm{E} t \wedge \delta[t / x])) \in \Delta$ for some $\mathbb{L}^{+}$-variable $t$. But this is impossible since $\Delta$ is $\mathbf{S}^{+}$-consistent.

As a result, for some $\mathbb{L}^{+}$-variable $z$, we have: $H(\wedge \Theta \wedge \Xi(\exists x \delta) \supset \neg \Xi(\exists z(\mathrm{E} z \wedge$ $\delta[z / x]))) \in \Delta$. But then $H \neg \wedge \Theta \in \Delta$. As a consequence, $\neg \wedge \Theta$ is in $\Omega$, and hence $\Gamma_{n}$ is $\mathbf{S}^{+}$-inconsistent. But by hypothesis, $\Gamma_{n}$ is $\mathbf{S}^{+}$-consistent.

Case 3.1 is treated as case 3.2 by letting $\Xi(\ldots)$ be the empty context, i.e. by letting $\Xi(X)$ just be $X$.

To each $\mathbf{S}^{+}$-nice set of $\mathbb{L}^{+}$-formulas $\Omega$ we associate a standard canonical model for $\mathbb{L}^{+}, M^{* \Omega}=\left\langle\mathscr{T}^{* \Omega},<^{* \Omega}, D^{* \Omega}, I^{* \Omega}\right\rangle$, whose construction goes as follows. Say that two $\mathbb{L}^{+}$-terms $m$ and $n$ are $\Omega$-equivalent iff $m=n \in \Omega$. By the reflexivity, symmetry and transitivity of $=$, the relation so defined is indeed an equivalence relation. We let $[\mathrm{m}]$ be the class of $\mathbb{L}^{+}$-terms which are $\Omega$-equivalent to $m$. We then lay down:

- $\mathscr{T}^{* \Omega}=$ the set consisting of $\Omega$ and all the $\mathbf{S}^{+}$-nice sets of $\mathbb{L}^{+}$-formulas $\Gamma$ such that $\Gamma<^{\mathbf{S}^{+}} \Omega$ or $\Omega<\mathbf{S}^{+} \Gamma$.

- $\Gamma<^{* \Omega} \Delta$ iff $\Gamma, \Delta \in \mathscr{T}^{* \Omega}$ and $\Gamma<^{\mathbf{S}^{+}} \Delta$.

- $D^{* \Omega}(\Delta)=\{[m]: \mathrm{E} m \in \Delta\}$.

- $I^{* \Omega}(a)=[a]$, and $\left\langle\left[m_{1}\right], \ldots,\left[m_{k}\right]\right\rangle \in I^{* \Omega}(\mathrm{F}, \Delta)$ iff $\mathrm{F} m_{1} \ldots m_{k} \in \Delta$, where $\mathrm{F}$ is a $k$-place predicate.

Given proposition 6.3, we then have:

\section{Proposition 6.5}

1. $<^{* \Omega}$ is transitive.

2. $<^{* \Omega}$ is total.

And given proposition 6.4:

Proposition 6.6 For all $\Delta \in \mathscr{T}^{* \Omega}$ and all $\mathbb{L}^{+}$-formulas $\phi$ :

1. $H \phi \in \Delta$ iff for all $\Gamma \in \mathscr{T}^{* \Omega}$ such that $\Gamma<^{* \Omega} \Delta, \phi \in \Gamma$.

2. $G \phi \in \Delta$ iff for all $\Gamma \in \mathscr{T}^{* \Omega}$ such that $\Delta<^{* \Omega} \Gamma, \phi \in \Gamma$.

Given proposition 6.5(2) and proposition 6.6 and the fact that $m=n \supset A(m=n)$ is a theorem of $\mathbf{S}^{+}$, we have: 
Proposition 6.7 For all $\Delta, \Gamma \in \mathscr{T}^{* \Omega}$ and $\mathbb{L}^{+}$-terms $m$ and $n$ : if $m=n \in \Delta$, then $m=n \in \Gamma$.

$\mathscr{T}^{* \Omega}$ is non-empty (it contains $\Omega$ ), and thanks to A14, proposition 6.6 and the fact that the members of $\mathscr{T}^{* \Omega}$ are $\mathbb{L}^{+}$-saturated, some $D^{* \Omega}(\Delta)$ is non-empty either. Given proposition 6.5 , then, $\left\langle\mathscr{T}^{* \Omega},<^{* \Omega}, D^{* \Omega}\right\rangle$ is a structure. Given $6.7, M^{* \Omega}$ is consequently indeed a model for $\mathbb{L}^{+}$. We can then prove the following by a standard induction on the complexity of the formulas:

Proposition 6.8 (Standard Truth Lemma) Let $r$ be the variable-assignment which assigns the value $[x]$ to each variable $x$ of $\mathbb{L}^{+}$. Then for all $\mathbb{L}^{+}$-formulas $\phi$ and $\Delta \in \mathscr{T}^{* \Omega}: \Delta \models_{r}^{M^{* \Omega}} \phi$ iff $\phi \in \Delta$.

The completeness of the neutral system with respect to the class of all models directly follows from the Standard Truth Lemma.

Proof The argument is standard. Set $\mathbf{S}=$ the neutral system formulated in $\mathbb{L}$. Suppose that $\mathbb{L}$-formula $\phi$ is not a theorem of $\mathbf{S}$, i.e. that $\{\neg \phi\}$ is $\mathbf{S}$-consistent. Let then $\Omega$ be an $\mathbf{S}^{+}$-nice extension of $\{\neg \phi\}$ in $\mathbb{L}^{+}$. Consider the model $M^{* \Omega}$ as constructed above, and let $r$ be as specified in the Lemma. Since $\neg \phi \in \Omega, \phi \notin \Omega$, and so by the Lemma, $\Omega \nvdash_{r}^{M^{* \Omega}} \phi$. This establishes that $\phi$ does not $\mathbb{L}^{+}$-hold in all models for $\mathbb{L}^{+}$. Now $M^{* \Omega}$ is a model for $\mathbb{L}^{+}$, but it is also a model for $\mathbb{L}$. Where $s$ is the restriction of $r$ to the $\mathbb{L}$-variables, we also have $\Omega \nvdash_{s}^{M^{* \Omega}} \phi$. Hence, $\phi$ does not $\mathbb{L}$-hold in all models for $\mathbb{L}$.

6.3 Completeness for Pres and Exp The previous construction is not suited to our aim, because the models $M^{* \Omega}$ we obtain via the construction are not based on proper structures. But we can hijack this construction to build suitable models. From here on, we assume that the base system $\mathbf{S}$ formulated in $\mathbb{L}$ is either Pres or Exp, and that $\mathbf{S}^{+}$is the same system but formulated in $\mathbb{L}^{+}$.

Consider a model for $\mathbb{L}^{+}, M^{* \Omega}=\left\langle\mathscr{T}^{* \Omega},<^{* \Omega}, D^{* \Omega}, I^{* \Omega}\right\rangle$, as constructed above. The main idea is to replace each member $\Delta$ of $\mathscr{T}^{* \Omega}$ by a distinguished member $t(\Delta)$ of $D^{* \Omega}(\Delta)$. Function $t$ is defined differently according to whether $\mathbf{S}$ is Pres or Exp. In the first case, we put: $t(\Delta)=[m]$, where $m$ is some $\mathbb{L}^{+}$-term such that $\mathrm{T} m \wedge \mathrm{E} m \in \Delta$. For any $\Delta \in \mathscr{T}^{* \Omega}$, AP1 guarantees that there is at least one term meeting the condition, and AP2 that any two terms meeting the condition are $\Omega$ equivalent; hence $t(\Delta)$ is well-defined. In the second case, we put: $t(\Delta)=[m]$, where $m$ is some $\mathbb{L}^{+}$-term such that $\mathrm{T} m \wedge \mathrm{E} m \wedge H \neg \mathrm{E} m \in \Delta$. That $t(\Delta)$ is well-defined is guaranteed this time by AE1 and AE2. (Given that AP3 belongs to the presentist system, the second definition of $t$ could also be used in the case of that system. Yet it is more perspicuous to adopt the proposed definition, and doing so will also be of some use in section 7.)

Proposition 6.9 $t$ is injective.

Proof (a) Case $\mathbf{S}=$ Pres. Consider $\Delta$ and $\Gamma$ in $\mathscr{T}^{* \Omega}$, and suppose $t(\Delta)=t(\Gamma)$. Then for some $\mathbb{L}^{+}$-term $m$, both $\mathrm{T} m$ and $\mathrm{E} m$ belong to both $\Delta$ and $\Gamma$. Now suppose for reductio that $\Delta \neq \Gamma$. Then since $<^{* \Omega}$ is total, $\Delta<^{* \Omega} \Gamma$ or $\Gamma<^{* \Omega} \Delta$. Let us only deal with the case $\Delta<^{* \Omega} \Gamma$, the other one being symmetrical. Since $T m, \mathrm{E} m \in \Gamma$, then by AP3, $H \neg \mathrm{E} m \in \Gamma$. But then by proposition 6.6(1), $\neg \mathrm{E} m \in \Delta$. But this is impossible since $\mathrm{E} m \in \Delta$ and $\Delta$ is Pres $^{+}$-consistent. (b) The case $\mathbf{S}=\mathbf{E x p}$ is treated in a very 
similar way (in this case AP3 need not be invoked, due to the definition of $t$ in the expansionist context).

Let us define the tuple $M^{\Omega}=\left\langle\mathscr{T}^{\Omega},<^{\Omega}, D^{\Omega}, I^{\Omega}\right\rangle$ as follows:

- $\mathscr{T}^{\Omega}=\left\{t(\Delta): \Delta \in \mathscr{T}^{* \Omega}\right\}$

- $t(\Gamma)<^{\Omega} t(\Delta)$ iff $\Gamma<^{* \Omega} \Delta$

- $D^{\Omega}(t(\Delta))=D^{* \Omega}(\Delta)$

- $I^{\Omega}(a)=I^{* \Omega}(a)$, and $\left\langle\left[m_{1}\right], \ldots,\left[m_{k}\right]\right\rangle \in I^{\Omega}(\mathrm{F}, t(\Delta))$ iff $\left\langle\left[m_{1}\right], \ldots,\left[m_{k}\right]\right\rangle \in I^{* \Omega}(\mathrm{F}, \Delta)$, where $\mathrm{F}$ is a $k$-place predicate

Given proposition $6.9, t$ is thus a bijection from $\mathscr{T}^{* \Omega}$ to $\mathscr{T}^{\Omega}$-it is indeed an isomorphism from $\left\langle\mathscr{T}^{* \Omega},<^{* \Omega}\right\rangle$ to $\left\langle\mathscr{T}^{\Omega},<^{\Omega}\right\rangle$. Since $M^{* \Omega}$ is a model for $\mathbb{L}^{+}$, so is $M^{\Omega}$, and it is clear that

Proposition 6.10 For all $\mathbb{L}^{+}$-formulas $\phi, \Delta \in \mathscr{T}^{* \Omega}$ and variable-assignments $r$ : $t(\Delta) \vDash_{r}^{M^{\Omega}} \phi$ iff $\Delta \models_{r}^{M^{* \Omega}} \phi$.

The Standard Truth Lemma then immediately yields another Truth Lemma:

Proposition 6.11 (New Truth Lemma) Let $r$ be the variable-assignment which assigns the value $[x]$ to each variable $x$ of $\mathbb{L}^{+}$. Then for all $\mathbb{L}^{+}$-formulas $\phi$ and $\Delta \in \mathscr{T}^{* \Omega}$ : $t(\Delta) \models_{r}^{M^{\Omega}} \phi$ iff $\phi \in \Delta$.

Using AT1 and proposition 6.6, one can show:

Proposition 6.12 For all $\Delta, \Gamma \in \mathscr{T}^{* \Omega}$ and $\mathbb{L}^{+}$-terms $m$ : if $T m \in \Delta$, then $T m \in \Gamma$.

This fact can be used to establish the following proposition:

Proposition 6.13 For all $\Delta \in \mathscr{T}^{* \Omega}$ and all $\mathbb{L}^{+}$-terms $m$ : T $m \in \Delta$ iff $[m] \in \mathscr{T}^{\Omega}$.

Proof (a) Suppose $\mathrm{T} m \in \Delta$. Due to A14, $S \mathrm{E} m \in \Delta$, and so by proposition 6.6, for some $\Gamma \in \mathscr{T}^{* \Omega}, \mathrm{E} m \in \Gamma$. Given proposition 6.12, we also have Tm$\in \Gamma$. If we assume $\mathbf{S}=$ Pres, then we have $[m]=t(\Gamma)$. Now suppose that we have $\mathbf{S}=\mathbf{E x p}$ instead. Given AE3, we then have: either $H \neg \mathrm{E} m \in \Gamma$ or $P(\mathrm{E} m \wedge H \neg \mathrm{E} m) \in \Gamma$. In the first case, $[m]=t(\Gamma)$. In the second case, proposition 6.6(1) guarantees the existence of some $\Lambda \in \mathscr{T}^{*} \Omega$ such that $\mathrm{E} m \wedge H \neg \mathrm{E} m \in \Lambda$. Given proposition 6.12, we also have $\mathrm{T} m \in \Lambda$. It follows that $[m]=t(\Lambda)$. (b) Conversely, suppose $[m] \in \mathscr{T}^{\Omega}$. This means that $[m]=t(\Gamma)$ for some $\Gamma \in \mathscr{T}^{* \Omega}$. But then $T m \in \Gamma$, and so by proposition 6.12, $\mathrm{T} m \in \Delta$.

In turn, proposition 6.13 can be used to establish the following fact:

Proposition 6.14 $\quad \mathscr{T}^{\Omega}$ has at least two members.

Proof $\quad$ By AT2, $S \exists x(\mathrm{~T} x \wedge S \exists y(\mathrm{~T} y \wedge x \neq y) \in \Omega$. By proposition 6.6, then, there are $\Delta$ and $\Gamma$ in $\mathscr{T}^{* \Omega}$ and $\mathbb{L}^{+}$-terms $m$ and $n$ such that: $T m \in \Delta$, Tn$\in \Gamma$ and $m \neq n \in \Gamma$. By propositions 6.13 and 6.7, it follows that $[m],[n] \in \mathscr{T}^{\Omega}$ and $[m] \neq[n]$.

Given proposition 6.14 and the fact that for all $t(\Delta) \in \mathscr{T}^{\Omega}, t(\Delta) \in D^{\Omega}(\Delta)$, the structure on which $M^{\Omega}$ is based is proper. That $M^{\Omega}$ is a proper model for $\mathbb{L}^{+}$then follows from proposition 6.13 .

We have:

Proposition 6.15 (1) If $\mathbf{S}=$ Pres, then $M^{\Omega}$ is a presentist model for $\mathbb{L}^{+}$. (2) If $\mathbf{S}=\mathbf{E x p}$, then $M^{\Omega}$ is an expansionist model for $\mathbb{L}^{+}$. 
Proof As we just saw, given any of the two systems, $M^{\Omega}$ is a proper model for $\mathbb{L}^{+}$. All we need to do here is prove that the underlying structure satisfies condition (c2), and that it satisfies (pres) if the system is Pres, and (exp) if the system is Exp (see the beginning of section 3 for the definition of the presentist and the expansionist structures).

(c2). Suppose for reductio that we have both $t(\Delta)<^{\Omega} t(\Gamma)$ and $t(\Gamma) \in D^{\Omega}(t(\Delta))$. Let $m$ be a term such that $t(\Gamma)=[m]$. We then have $H \neg \mathrm{E} m \in \Gamma$ (by definition if we work with the expansionist system, due to AP3 if we work with the presentist system). Since $t(\Gamma) \in D^{\Omega}(t(\Delta))$, E $m \in \Delta$. Now given that $t(\Delta)<^{\Omega} t(\Gamma)$, we have $\Delta<^{* \Omega} \Gamma$, and so by proposition 6.6(1) we get $\neg \mathrm{E} m \in \Delta$. But this is impossible since $\Delta$ is $\mathbf{S}^{+}$-consistent.

(pres). Assume $\mathbf{S}=$ Pres. Suppose for reductio that we have both $t(\Gamma)<^{\Omega} t(\Delta)$ and $t(\Gamma) \in D^{\Omega}(t(\Delta))$. Let $m$ be a term such that $t(\Gamma)=[m]$. We then have $\mathrm{T} m \wedge \mathrm{E} m \in \Gamma$. Note that due to the presence of AT1 and AP3, $\forall x(\mathrm{~T} x \supset G \neg \mathrm{E} x)$ is a theorem of Pres. We accordingly also have $G \neg \mathrm{E} m \in \Gamma$. Now we can reason as in the case of condition (ii). Since $t(\Gamma)<^{\Omega} t(\Delta)$, we have $\Gamma<^{* \Omega} \Delta$, and so by proposition 6.6(2) we get $\neg \mathrm{E} m \in \Delta$. But since $t(\Gamma) \in D^{\Omega}(t(\Delta))$, E $m \in \Delta$. This is impossible since $\Delta$ is Pres $^{+}$-consistent.

(exp). Assume $\mathbf{S}=\mathbf{E x p}$. Assume $t(\Delta)<^{\Omega} t(\Gamma)$. Then $\Delta<^{* \Omega} \Gamma$. Let $[m] \in D^{\Omega}(t(\Delta))$. Then $\mathrm{E} m \in \Delta$. By AE4 it follows that $G \mathrm{E} m \in \Delta$. Given that $\Delta<^{* \Omega} \Gamma$ and given proposition 6.6(2), we infer $\mathrm{E} m \in \Gamma$, and hence $[m] \in D^{\Omega}(t(\Gamma))$.

Completeness is then immediate:

Theorem 6.16 (Completeness) (1) An $\mathbb{L}$-formula is a theorem of Pres if it $\mathbb{L}$-holds in every presentist model for $\mathbb{L}$. (2) An $\mathbb{L}$-formula is a theorem of $\mathbf{E x p}$ if it $\mathbb{L}$-holds in every expansionist model for $\mathbb{L}$.

Proof Let $\phi$ be an $\mathbb{L}$-formula that is not a theorem of Pres. Then $\{\neg \phi\}$ is Presconsistent. Let $\Omega$ be a Pres ${ }^{+}$-nice extension of $\{\neg \phi\}$ in $\mathbb{L}^{+}$, and let the model $M^{\Omega}=\left\langle\mathscr{T}^{\Omega},<^{\Omega}, D^{\Omega}, L^{\Omega}, I^{\Omega}\right\rangle$ be as defined above, with $\mathbf{S}=$ Pres. By proposition 6.15(1), $M^{\Omega}$ is a presentist model for $\mathbb{L}^{+}$, and hence for $\mathbb{L}$. Since $\neg \phi \in \Omega, \phi \notin \Omega$, and so by the New Truth Lemma we get $t(\Omega) \not \nvdash_{r}^{M^{\Omega}} \phi$. If $s$ is the restriction of $r$ to the $\mathbb{L}$-variables, we then have $t(\Omega) \nvdash_{s}^{M^{\Omega}} \phi$. Hence, $\phi$ does not $\mathbb{L}$-hold in $M^{\Omega}$. (2) is established in the very same way, but using proposition 6.15(2) instead of proposition $6.15(1)$.

\section{Variants}

Here we describe some variants of the systems introduced above. Throughout this section, we suppose given a fixed temporal language, which we do not bother to mention.

7.1 An alternative to AT1 Some readers may object to axiom AT1, i.e. $T x \supset A \top x$, on the grounds that being a time entails existing: if being a time entails existing, then a consequence of AT1 is that every time always exists, and this goes against both presentism and expansionism. We are not impressed by this argument (why should we believe that being a time is existence-entailing?), but we do not want to argue the case in the present context: our characterisation of presentism and expansionism can accommodate the view that being a time is existence-entailing. 
On the proof-theoretic side, the idea is to replace AT1 in both Pres and Exp by the following axioms:

AT1a: $\mathrm{T} x \supset \mathrm{E} x$

AT1b: $\mathrm{T} x \supset A(\mathrm{E} x \supset \mathrm{T} x)$

On the semantic side, the modification consists in replacing the condition on the interpretation of $\mathrm{T}$ in proper models by the following condition:

- For all $t \in \mathscr{T},\langle o\rangle \in I(\mathrm{~T}, t)$ iff both $o \in \mathscr{T}$ and $o \in D(t)$.

Given this condition, both AT1a and AT1b hold in every proper model. This secures the soundness of the new systems. Assuming that the systems have AT1a and AT1b rather than AT1, propositions 6.12 and 6.13 are false. Instead of proposition 6.12, we have:

- For all $\Delta, \Gamma \in \mathscr{T}^{* \Omega}$ : if $\mathrm{T} m \in \Delta$, then $\mathrm{T} m \in \Gamma$ provided that $\mathrm{E} m \in \Gamma$,

and instead of proposition 6.13, we have:

- For all $\Delta \in \mathscr{T}^{* \Omega}$ and all terms $m$ of the extended language: $T m \in \Delta$ iff both $[m] \in \mathscr{T}^{\Omega}$ and $\mathrm{E} m \in \Delta$.

This latter proposition, just like proposition 6.13, guarantees that the constructed model $M^{\Omega}$ is a proper model for the extended language. Proposition 6.15 still holds given the new systems (AT1 is invoked in the proof of proposition 6.15(1), as helping secure that $\forall x(\mathrm{~T} x \supset G \neg \mathrm{E} x)$ is a theorem of the presentist system, but ATlb is in fact strong enough in the context). The completeness of the new systems then follows.

7.2 Presentism with times that precede themselves It should be clear that expansionism is inconsistent with the view that some time may be earlier than itself: for given expansionism, what exists at a time strictly includes what exists at any earlier time, and strict inclusion is irreflexive. By contrast, on the face of it, presentism is consistent with the view that there are times preceding themselves, in particular with the view that time is circular. Yet our proposed logic for presentism precludes such views. Views that entail that some times precede themselves are certainly exotic, and it may therefore be thought that it is not problematic to discard them. However, we would like to show that the previous material can easily be modified to leave room for such views.

Semantically, the idea is to replace conditions (c2) and (pres) in the definition of presentist structures by the following single condition:

(w-pres): For all $u, v \in \mathscr{T}, u \in D(v) \Rightarrow u=v$.

Call the new presentist structures / models weak presentist structures / models. A structure / model is presentist iff it is weakly presentist and its precedence relation is irreflexive. An example of a non-irreflexive weakly presentist structure is provided by any structure $\langle\mathscr{T},<, D\rangle$ where $<$ is universal on $\mathscr{T}$ and $D(u)=\{u\}$ for any $u \in \mathscr{T}$.

On the proof-theoretic side, the suggestion is to replace AP3 by the schema

AP4: $\forall x(\mathrm{~T} x \wedge \phi \supset A(\mathrm{E} x \supset \phi))$

Call the resulting system $\boldsymbol{w}$-Pres. AP4 holds in all weak presentist models, and so wPres is sound with respect to the modified presentist semantics. AP3 fails to hold in weak presentist models with times that precede themselves. Thus, w-Pres is strictly included in Pres. 
In order to establish completeness, a bit more work is required. The definition of function $t$ in the completeness proof is the same as for the original presentist system. But the proof that $t$ is injective must be modified. Here is an appropriate proof in the present context:

- Consider $\Delta$ and $\Gamma$ in $\mathscr{T}^{* \Omega}$, and suppose $t(\Delta)=t(\Gamma)$. Then for some $\mathbb{L}^{+}$-term $m$, both $T m$ and $\mathrm{E} m$ belong to both $\Delta$ and $\Gamma$. Let $\phi$ be an arbitrary $\mathbb{L}^{+}$-formula such that $\phi \in \Delta$. By AP4, then, $A(\mathrm{E} x \supset \phi) \in \Delta$, and so by proposition 6.6, $\phi \in \Gamma$. Thus, $\Delta \subseteq \Gamma$. By symmetry, we also have the converse, and hence $\Delta=\Gamma$.

The only other place in the completeness proof for Pres where AP3 plays a role is the proof of proposition 6.15(1). What should be established in the present context is that if the base system is w-Pres, then $M^{\Omega}$ is a weak presentist model for $\mathbb{L}^{+}$. What has to be proved in order to get this is the following:

- For all $t(\Delta), t(\Gamma) \in \mathscr{T}^{\Omega}, t(\Delta) \in D^{\Omega}(t(\Gamma)) \Rightarrow t(\Delta)=t(\Gamma)$.

Here is a proof:

- Let $t(\Delta)$ be $[m]$ and $t(\Gamma)$ be $[n]$. Assume $t(\Delta) \in D^{\Omega}(t(\Gamma))$. Then thanks to AT1 (in fact, AT1b is already enough) and proposition 6.6, we then have $\mathrm{T} m, \mathrm{~T} n, \mathrm{E} m, \mathrm{E} n \in \Gamma$. But then by AP2, $[m]=[n]$, i.e. $t(\Delta)=t(\Gamma)$.

With this in place, the completeness of $\mathbf{w}$-Pres follows.

It should be clear that soundness and completeness are preserved if we replace AT1 by AT1a and AT1b in the system, and make the corresponding modification in the semantics that is described in the previous section.

\section{Principles for location}

Here we deal with the issue of formulating general principles governing the temporal location predicate L. Here is a set of axioms that we find natural, whatever version of temporaryism is accepted in the first place:

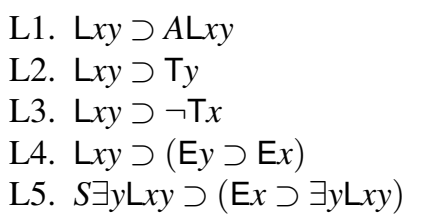

Once L1-L5 are added to any of the systems discussed so far, soundness and completeness will be preserved by requiring that the interpretation functions of the models satisfy the conditions encoded in L1-L5. We leave the details aside.

The only axiom in the list that is unquestionable is L2. L1 may be rejected on grounds similar to those motivating rejection of AT1 that we discussed in the previous section. L3 sounds compulsory on a conception of times as "containers" in which entities such as continuants and occurents find their place. But if we view times as maximal fusions of contemporary instantaneous events, and we take fusions of temporally located entities to be themselves temporally located entities, then L3 will have to be given up.

Rejecting L4 or L5 may initially look difficult. Granted that Plato's birth is located at time $t$, how could it ever be both true that $t$ exists and false that Plato's birth exists? Thus prima facie, L4 seems solid. It is especially hard to see how L5 could be rejected, given the key ontological consequences it has in the present context. The 
condition on $x$ in its antecedent marks out the set of "temporal inhabitants", and L5 says that no object from that category can exist without being temporally located. Combined with Pres, w-Pres or Exp (or the variants obtained by replacing AT1 by AT1a and AT1b), L5 yields desirable consequences. Let $\pi$ be the present time (the time at which we are speaking now). Then an object is present iff it is located at $\pi$, and an object is merely past (future) iff it is located at a time that precedes (succeeds) $\pi$ but not at $\pi$ itself. On both Pres and w-Pres (and also on the variants that have AT1a and AT1b instead of AT1), $\pi$ is the unique time that there is, and by L5, then, every temporal inhabitant is present, and no temporal inhabitant is merely past or merely future. This sounds as it should be. On Exp (and the corresponding variant), no time succeeds $\pi$, and therefore, by L5, every temporal inhabitant is either present or not merely future. Granted that sometimes in the past, there were times occupied by (say) Socrates and events involving Socrates, then given Exp, there are times preceding $\pi$, and temporal inhabitants that are merely past. This again sounds as it should be.

However, given certain ontological assumptions, counterexamples to L4 and L5 can be provided (see Correia and Rosenkranz 2018, §3.2). Assume that a set exists only when all its members do, and that a set is temporally located whenever at least one of its members is located. On these assumptions, and given either presentism or expansionism, at the time $t$ of Plato's birth, the set \{Plato's birth, Hume's birth $\}$ is located at $t$ but does not exist, while $t$ itself does exist-contrary to what L4 entails. Assume that a fusion exists whenever at least one of the fused objects exists, and that a fusion is located at a time only iff at least one of the fused objects is located at that time. Consider then the fusion of Hume's birth and the number 7. It is located whenever Hume's birth is located, and hence it is sometimes located. By L5, it should then be located whenever it exists. But given presentism or expansionism and the extra assumption that numbers always exist, this is not the case.

These admittedly somewhat exotic counterexamples rely on substantive metaphysical assumptions that would need to be properly assessed, of course.

\section{Correia and Rosenkranz, 2013 and 2015}

Let us here compare the characterisations of presentism and expansionism put forward in this paper with the characterisation of presentism we advocated in Correia and Rosenkranz 2015 (hereafter: the 2015 characterisation) and the characterisation of expansionism we defended in Correia and Rosenkranz 2013 (hereafter: the 2013 characterisation).

The 2015 characterisation can be expressed in temporal languages as follows:

$$
A \exists x(\mathrm{~T} x \wedge H \neg \mathrm{E} x \wedge G \neg \mathrm{E} x \wedge \forall y((\mathrm{~T} y \supset y=x) \wedge(S \exists z \operatorname{L} y z \supset \operatorname{L} y x)))
$$

Assuming the neutral system (only minimal tense logic and the proposed quantification theory are actually enough), this characterisation is equivalent to the conjunction of the presentist axioms of Pres, namely AP1, AP2, AP3, and of axiom L5 for temporal location. Hence, Pres + L5 can be seen as a systematisation of the non-systematic characterisation of presentism offered in the 2015 paper. As we have argued in the previous section, presentism seems to be compatible with the rejection of L5. Thus, reliance on L5 makes the 2015 characterisation unsatisfactory-that is, until the ontological assumptions on which the counterexample to presentism + L5 were based have been shown to be untenable. 
The 2013 characterisation is not as straightforwardly connected to Exp, for a number of reasons: it makes use of so-called metric temporal operators and of a predicate for presentness, which are foreign to temporal languages as we have defined them, it does not state any specific principles about times, and it makes the simplifying assumption that things in time are instantaneous, an assumption which is acceptable given the dialectics of Correia and Rosenkranz 2013 but which we did not take on board in the present context (and rightly so, since obviously, the content of the assumption is not built into expansionism). However, the 2013 characterisation and Exp share a common spirit.

Given expansionism, there is a notion which is definable in all temporal languages and whose extension is always identical to the extension of the notion of being $a$ present time (as the latter is usually understood), to wit the notion of being a time that exists but never existed before (see section 4), i.e. the notion of being a time that is new (see the end of section 5 for the definition of 'is new'). Suppose, then, that we understand 'is present' as 'is new'. According to the 2013 characterisation, expansionism is the view that three principles always hold. These three principles can be formulated as follows (we here keep the original numbering):

(1) Everything either is present or was present in the past.

(2) For any positive number $n$, what there was $n$ days ago still exists $n$ days later (i.e. now).

(3) Something is present, and for any positive number $n$, there is something that was present $n$ days ago.

Given the proposed interpretation of the presentness predicate, axiom AE3* is just (1) but restricted to times. (Unrestricted (1) has to be rejected, we take it, because it rules out the possibility of sempiternal entities, with which expansionism per se should be compatible.) Given plausible assumptions about the relations between the metric operators and the standard Priorean operators, the claim that (2) always holds is equivalent to the claim that AE4 always holds. Finally, again given plausible assumptions, in the presence of (2), (3) is equivalent to the following principle:

$\left(3^{*}\right)$ Presently as well as always in the past, something is present.

Assuming the proposed interpretation of 'is present', $\left(3^{*}\right)$ states that presently as well as always in the past, something is new. In the neutral system, the claim that this always holds is equivalent to the claim that always, something is new. The claim that $\mathrm{AE}^{*}$ always holds is just this latter claim but with 'something' being restricted to times.

\section{Correia and Rosenkranz 2018}

Let us finally compare the logics for presentism and expansionism put forward in this paper with those we gave in Correia and Rosenkranz 2018 (see in particular Appendix 1 for self-contained material).

Correia and Rosenkranz 2018 introduces just one logic for presentism and one for expansionism. The common language of these logics is a temporal language (see section 2) enriched with both a predicate for precedence between times and an operator @ for truth-at-a-time. From a proof-theoretic point of view, both logics have a common core, a "neutral system" that includes our neutral system (see section 5) but has in addition axioms for the truth-at-a-time operator and the extra-logical predicates (namely the predicates for times, temporal location and precedence between times), 
and each is defined by that core plus a set of specific axioms. For expansionism, the axioms are

P1: Ex $\supset G \mathrm{E} x$ (which amounts to our EA4 given the background logic of quantification)

P2: $\mathrm{T} x \supset @_{x} H \neg \mathrm{E} x$

and for presentism they are

P2: $\mathrm{T} x \supset @_{x} H \neg \mathrm{E} x$

P3: $\mathrm{T} x \supset @_{x} G \neg \mathrm{E} x$

The models for presentism and those for expansionism are, cosmetic details aside, the same as the models introduced in this paper but with one important difference: in each type of model, the precedence relation is required to be irreflexive.

This latter point could be used to argue against the characterisation of presentism provided in Correia and Rosenkranz 2018 (see section 7.2), but there is a much more powerful argument in favour of the characterisations of both views that we put forward in this paper. What our study shows is that both presentism and expansionism can be characterised in a language that is extremely lean by the standards of temporaryism: a first-order language with identity, enriched with a pair of simple Priorean operators, a predicate for times and a predicate for temporal location. As we advertised in section 1, there is no need for a presentness predicate, let alone for two predicates expressing distinct notions of presentness, for predicates that are neither tensed nor non-tensed, or for non-standard operators of the sorts mentioned in Sider 2001 and Tooley 1997. As we have established here, there is no need either for a truth-at-a-time operator, contrary to what the results we established in Correia and Rosenkranz 2018 would seem to suggest. ${ }^{7}$

\section{Notes}

1. According to a far less prominent, but nonetheless noteworthy view, which we might call the shrinking block theory of time, everything in time is present or will be present, and something in time is not present (cf. Dummett 2004).

2. The qualification between brackets, which will be mostly left implicit in what follows, is important since it is not our intention to provide characterisations that fit all possible, or even all extant, conceptions of presentism and expansionism.

3. Our characterisations of presentist views should also refute the recently advanced claim that presentism either is trivial or inadvertently collapses into permanentism (see e.g. Crisp 2004; Meyer 2005 and 2013; for further references, see Ingram and Tallant 2018). Elsewhere we have argued that this claim is likely to rest on a confusion between temporal existence and temporal location (Correia and Rosenkranz forthcoming; see also Correia and Rosenkranz 2015 and 2018).

4. At least one prominent proponent of presentism, Arthur N. Prior, would take the extension of T, as thus understood, to be always empty: Prior opposes time substantivalism, dislikes events, and is indeed known for defending an ersatzist conception of time instants (see e.g. Prior 1967, pp. 74-76 and Prior 1968). Other presentists do not follow Prior in this regard, but are happy to postulate that there always is a concrete time (Markosian 2004: 76; cf. also Correia and Rosenkranz 2015). This discrepancy is not a problem for us since, 
as we emphasised earlier (footnote 2), our aim is not to be faithful to all possible versions of presentism. Likewise, the fact that there may be disagreement among expansionists about the existence of concrete times need not bother us, for the same reason.

5. The formulations advocated in Correia and Rosenkranz 2018 are of that sort; see section 10 below.

6. See Hugues and Cresswell 1996, pp. 296-302, for the case of a modal logic that is relevantly similar.

7. It is also worth noting that the completeness proofs in Correia and Rosenkranz 2018 are very different from those we provide here. They make essential use of the fact that the object language contains a predicate for precedence and a truth-at-a-time operator, and, unlike ours, they do not proceed via the standard construction that we develop in section 6.2 .

\section{References}

Bourne, C. 2002. When am I? A Tense Time for Some Tense Theorists?, Australasian Journal of Philosophy 80, 359-71.

Braddon-Mitchell, D. 2004. How do we know that it is now now?, Analysis 64, 199-203.

Button, T. 2006. There's no time like the present, Analysis 66, 130-35.

Button, T. 2007. Every Now and Then, no-futurism faces no sceptical problems, Analysis 67, 325-32.

Correia, F. and Rosenkranz, S. 2013. Living on the Brink, or Welcome Back, Growing Block!, Oxford Studies in Metaphysics, 8, 333-50.

Correia, F. and Rosenkranz, S. 2015. Presentism without Presentness, Thought: A Journal of Philosophy 4, 19-27.

Correia, F. and Rosenkranz, S. 2018. Nothing To Come: A Defence of the Growing Block Theory of Time, Synthese Library 395, New York: Springer.

Correia, F. and Rosenkranz, S. forthcoming. Temporal Existence and Temporal Location, Philosophical Studies.

Crisp, T. M. 2004. On Presentism and Triviality, in D. W. Zimmerman (ed.), Oxford Studies in Metaphysics 1, Oxford: Oxford University Press, 15-20.

Dummett, M. 2004. Truth and the Past, New York: Columbia University Press.

Hughes, G.E. and Cresswell, M. J. 1996. A New Introduction to Modal Logic, London and New York: Routledge.

Ingram, D. and Tallant, J. 2018. Presentism, in E. N. Zalta (ed.), The Stanford Encyclopedia of Philosophy <https://plato.stanford.edu/archives/spr2018/entries/presentism/>.

Markosian, N. 2004. A Defense of Presentism, in D. W. Zimmerman (ed.), Oxford Studies in Metaphysics 1, Oxford: Oxford University Press, 47-82.

Merricks, T. 2006. Goodbye Growing Block, Oxford Studies in Metaphysics 2, 103-10.

Meyer, U. 2005. The Presentist's Dilemma, Philosophical Studies, 122, 213-225. 
Meyer, U. 2013. The Triviality of Presentism, in R. Ciuni et al. (eds.), New Papers on the Present: Focus on Presentism, Munich: Philosophia Verlag, 67-87.

Prior, A. N. 1967. Past, Present, and Future, Oxford: Oxford University Press.

Prior, A. N. 1968. Changes in Events and Changes in Things, in Papers on Time and Tense, Oxford: Oxford University Press, 1-15.

Sider, T. 2001. Four-Dimensionalism, Oxford: Oxford University Press.

Tooley, M. 1997. Time, Tense and Causation, Oxford: Oxford University Press.

Williamson, T. 2013. Modal Logic as Metaphysics, Oxford: Oxford University Press.

\title{
Acknowledgments
}

The research that led to this publication was funded by the European Commission's H2020 programme under grant agreement H2020-MSCA-ITN-2015-675415, and by the Swiss National Science Foundation though project BSCGI0-157792.

\author{
Correia \\ Department of Philosophy \\ University of Geneva \\ Rue De-Candolle 5 \\ 1211 Geneva 4 \\ SWITZERLAND \\ fabrice.correia@unige.ch \\ Rosenkranz \\ ICREA \\ Pg Lluís Companys 23 \\ 08010 Barcelona \\ SPAIN \\ Department of Philosophy \\ University of Barcelona \\ Montalegre 6-8 \\ 08001 Barcelona \\ SPAIN \\ rosenkranz.sven@gmail.com
}

